\title{
Pityriasis Alba versus Vitiligo Clinical and Histopathological Study
}

\author{
Khalifa E. Sharquie ${ }^{1,2^{*}}$, Adil A. Noaimi1,2, Liqaa M. Abdulkadhim ${ }^{3}$ \\ ${ }^{1}$ Department of Dermatology, College of Medicine, University of Baghdad, Baghdad, Iraq \\ ${ }^{2}$ Iraqi and Arab Board for Dermatology and Venereology, Baghdad Teaching Hospital, Baghdad, Iraq \\ ${ }^{3}$ Department of Dermatology, Baghdad Teaching Hospital, Baghdad, Iraq \\ Email: *ksharquie@ymail.com, adilnoaimi@yahoo.com, liqaamohammed96@gmail.com
}

How to cite this paper: Sharquie, K.E., Noaimi, A.A. and Abdulkadhim, L.M. (2018) Pityriasis Alba versus Vitiligo Clinical and Histopathological Study. Journal of Cosmetics, Dermatological Sciences and Applications, 8, 140-150.

https://doi.org/10.4236/jcdsa.2018.83016

Received: December 11, 2017

Accepted: September 23, 2018

Published: September 26, 2018

Copyright (C) 2018 by authors and Scientific Research Publishing Inc. This work is licensed under the Creative Commons Attribution International License (CC BY 4.0).

http://creativecommons.org/licenses/by/4.0/

\section{Open Access}

\begin{abstract}
Background: Most recently there was a well established study showed that pityriasis alba of ten progresses into vitiligo (Sharquie et al.). These findings were considered a new thought that might support a new hypothesis regarding the pathogenesis of vitiligo. Objective: In order to do further work on hypothesis which is in favor that pityriasis alba might progress into vitiligo, so the present research including clinical and histopathological study to confirm that pityriasis alba might progress to vitiligo. Patients and method: This is a descriptive clinical and histopathological comparative study carried out in Department of Dermatology, Baghdad Teaching Hospital during the period from January 2015 to August 2016. Thirty eight patients with pityriasis alba (group A) and twenty eight patients with vitiligo stage I (group B) and twelve patients with both pityriasis alba and vitiligo (group C) were included in this study. All patients were fully interviewed and full history was taken from all the patients. Also careful asking about personal and family history of vitiligo was taken. Blunt trauma was done to induce Koebner's phenomenon. Punch biopsies were taken from skin lesion of all patients and ten biopsies from 10 persons were taken from normal skin. Each biopsy specimen was processed and stained with Hematoxylin-eosin ( $\mathrm{H} \& \mathrm{E}$ ) and Fontana-Masson (FM) for histological evaluations. Results: Clinical study-Group A: Pityriasis alba patients: The mean of age of patients in pityriasis alba \pm SD was $8.68 \pm 5.94$ years including $33(86.84 \%)$ males and $5(13.16 \%)$ females. Koebner's phenomenon was positive in $2(5.26 \%)$ patients and family history of vitiligo was positive in $17(44.74 \%)$ patients. Group B: stage I vitiligo patients: The mean of age \pm SD was $15.46 \pm 12.50$ years with 15 (57.14\%) male patients and $12(42.86 \%)$ female patients. A Koebner's phenomenon was positive in 11 (39.28\%) patients and family history of vitiligo was found in 11 (39.29\%) patients. Group C: pityriasis alba and vitiligo patients: The mean of age \pm SD
\end{abstract}


was $8.33 \pm 2.78$ years including 9 (75\%) male patients and $3(25 \%)$ female patients. Koebner's phenomenon was positive in $6(50 \%)$ patients and family history of vitiligo was positive in 7 (58.33\%) patients. Histopathological study: Group A: pityriasisalba: Hematoxylin-Eosin (H \& E) stain: Regarding melanin stores, basal melanin was found to be decreased when compared with normal skin in $23(60.53 \%)$ patients. Fontana Masson stain: showed decrease in basal melanin when compared with normal skin in 24 (63.16\%) patients. Group B: stage I vitiligo patients: Hematoxylin-Eosin ( $\mathrm{H} \& \mathrm{E})$ stain: Partial basal melanin decrease was detected in $19(67.86 \%)$ patients while Fontana stain showed Partial melanin decrease in 20 (71.43\%) patients. Group C: combination pityriasis alba and vitiligo: In vitiligo lesion, $\mathrm{He}-$ matoxylin-Eosin ( $\mathrm{H} \& \mathrm{E}$ ) stain: Basal melanin was decreased in 6 (50\%) patients as compared with normal skin. Fontana stain: showed reduction in basal melanin in $8(66.67 \%)$ patients. In pityriasis alba: Hematoxylin-Eosin (H \& E) stain: Basal melanin was partially decreased in $8(66.67 \%)$ patients while Fontana stain showed partial decrease of melanin in $6(50 \%)$ patients. Regarding the inflammatory reaction was almost similarly observed in three groups in a form of dermal superficial lymphocytic reaction and lymphocytic epidermal invasion. Conclusion: The present study strongly supported the hypothesis that said pityriasis alba and vitiligo could be part of one spectrum as they shared strongly positive family history of vitiligo and high Koebner's phenomenon in all three groups. Also there were many histopathological similarities in three groups including pigments loss and inflammatory reaction in epidermis and dermis.

\section{Keywords}

Pityriasis Alba, Vitiligo, Comparison

\section{Introduction}

Pityriasis alba (PA) is a common benign condition mainly affecting the head and neck regions of preadolescent children, more noticeable in darker skin types and there is no gender or skin type predilection [1] occurs predominantly in children between the ages of 3 and 16 years [2]. The condition is more frequently seen in children and adolescents than in adults, it has been reported in $40 \%$ of Egyptian and $38.22 \%$ of Iraqi children [3], but is more common among males in Iraq [3]. The lesions are frequently limited to the face, but the neck, arms, and shoulders can also be affected [3]. The etiology remains not well elucidated but pityriasis alba has been commonly characterized as a mild form of atopic dermatitis [1]. Excessive, unprotected sun exposure as well as hygienic habits (frequent bathing and hot baths) are strongly related to the development of pityriasis alba [1]. All these conditions present as whitish scaly patches with ill-defined border (Sharquie personal communication 2013). Accordingly, we are facing a great challenge regarding distinction criteria of differentiation between pityriasis alba 
from early vitiligo or whether pityriasis alba progresses to vitiligo, or other post-inflammatory hypopigmentation. The criteria for distinction of pityriasis alba from early vitiligo include scaly lesion, indistinct margin, positive or negative wood's lamp and negative family history of vitiligo [4].

Accordingly we wonder whether pityriasis alba is clinical entity or part of manifestations of other inflammatory skin diseases, it could be part of atopic dermatitis and sometime psoriasis might present with pityriasis alba on face or could be part of dry skin or as manifestation of vitiligo [1].

In a recent study by Sharquie et al., $43.75 \%$ of the patients with pityriasis alba had progressed into ordinary vitiligo and this was confirmed by high positive family history of vitiligo, positive Koebner's phenomenon and complete absence of melanin by histopathological study [4].

Accordingly there is a high risk factor among patients with PA to change into vitiligo as both PA and vitiligo are inflammatory hypopigmented skin diseases [5] [6] [7].

\section{Patients and Methods}

A case descriptive, clinical and histopathological comparative study was carried out in the Department of Dermatology, Baghdad Teaching Hospital, Baghdad, Iraq, during the period from January 2015 to August 2016.

Thirty eight patients with pityriasis alba and twenty eight patients with stage I vitiligo and twelve patients with both pityriasis alba and vitiligo were included in this study.

All patients were fully interviewed and full history was taken from all the patients regarding the time of onset and the progression of the disease, and regarding pityriasis alba also asking about history of sun exposure and seasonal variation and history of excessive bathing and careful asking about family history of vitiligo. Physical examination was carried out regarding the clinical appearance of the patches, the site of the lesions, edges, size and the degree of pigmentation. This was confirmed by Wood's lamp to assess the degree of pigmentation (as confirmatory test). Blunt trauma to induce Koebner's phenomenon was done for all patients on the hidden area (back or upper arm) by blunt object, and watched after 1 week and after 1 month to see the appearance of leukoderma.

Punch biopsies were taken from skin lesion in the face or arms for all patients using sterilized stainless steel punch biopsy and ten biopsies from 10 person were taken from normal skin and processed similar to the patients biopsies. Each biopsy specimen was processed and stained with Hematoxylin-eosin ( $\mathrm{H} \& \mathrm{E})$ and Fontana-Masson (FM) stain was used for histochemical evaluations. Ensuring a formal consent of the parents of the patients and full explanation about the nature of disease, course, follow up and before taking photograph and biopsy and the ethical approval was obtained from the Scientific Council of Dermatology \& Venereology-Iraqi Board for Medical Specializations.

Statistical comparison between two groups was performed using chi square test. 


\section{Results}

Thirty eight patients with pityriasis alba (group A) and twenty eight patients with vitiligo (group B) and twelve patients with both pityriasis alba and vitiligo in the same patient (group C) were included in this study.

\subsection{Clinical Study}

Group A; (Pityriasis alba) The mean of age \pm SD was $8.68 \pm 5.94$ years including $33(86.84 \%)$ males and $5(13.16 \%)$ females. The duration of disease less than 1 year was $20(52.63 \%)$ patients and from $1-5$ years was $17(44.74 \%)$ patients and more than 5 year was $1(2.63 \%)$ patient.

The type lesions were erythematous scaly plaques in 7 (18.42\%) patients and hypochromicscaly plaques in $22(57.89 \%)$ patients and smooth hypochromic in 9 (23.68\%) patients (Figure 1). While Koebner's phenomenon was positive in 2 (5.26\%) patients (Figure 2).

Personal history of atopy was positive in $14(36.84 \%)$ patients and family history of atopy was positive in 18 (50\%) patients while family history of vitiligo was positive in $17(44.74 \%)$ patients. Family history of other autoimmune disease was positive in $3(7.89 \%)$ (Table 1$)$.

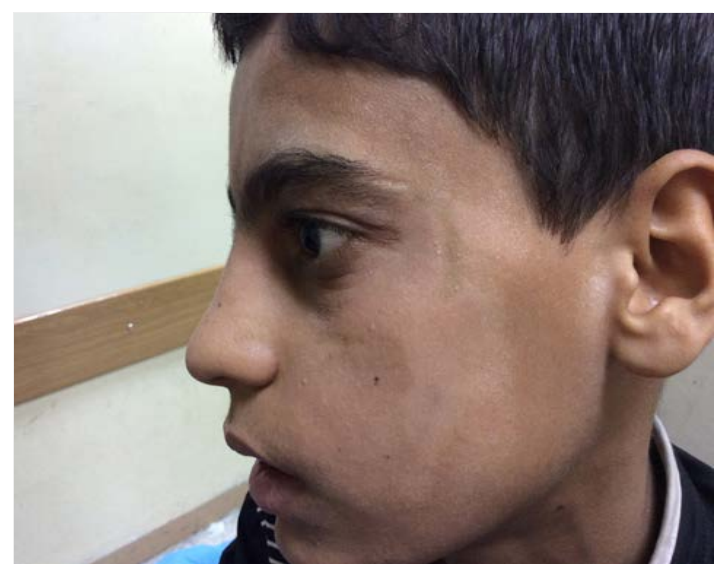

Figure 1. Fifteen years old male with severe Pityriasis alba lesion on the face for 2 years.

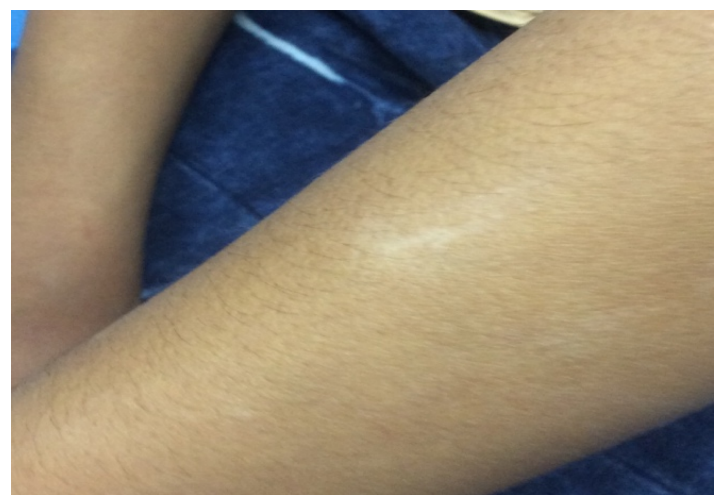

Figure 2. Fourteen years old male with Pityriasis alba for 8 months with positive Koebner's phenomenon. 
Table 1. Demographic data for all patients of three groups.

\begin{tabular}{cccc}
\hline Demographic data & $\begin{array}{c}\text { Pityriasis } \\
\text { alba (group A) }\end{array}$ & $\begin{array}{c}\text { Vitiligo } \\
\text { (group B) }\end{array}$ & $\begin{array}{c}\text { Pityriasis alb and } \\
\text { Vitiligo (group C) }\end{array}$ \\
\hline Numberof patients & 38 & 28 & 12 \\
Male Female & $33(86.89 \%)$ & $16(57.14 \%)$ & $9(75 \%)$ \\
Age (mean \pm SD) & $5(13.16 \%)$ & $12(42.86 \%)$ & $3(25 \%)$ \\
& $8.68 \pm 5.94$ Years & $15.46 \pm 12.50$ years & $8.33 \pm 2.78$ years \\
Duration of disease & $20(52.89 \%)$ & $14(50 \%)$ & In PA \\
Less than 1 year & $17(44.74 \%)$ & $10(35.71 \%)$ & $6(50 \%)$ \\
1 - 5 years & $1(2.63 \%)$ & $4(14.29 \%)$ & $3(25 \%)$ \\
More than 5 years & & & $<1$ year $9(75 \%)$ \\
& & $11(3.57 \%)$ & $1-5$ years $3(25 \%)$ \\
Family history of atopy & $19(50 \%)$ & & $5(41.67 \%)$ \\
Family history of vitiligo & $17(44.74 \%)$ & $11(39.29 \%)$ & $7(58.33 \%)$ \\
Family history of other & $3(7.89 \%)$ & $8(28.57 \%)$ & $5(41.67 \%)$ \\
autoimmune disease & $14(36.84 \%)$ & $6(21.43 \%)$ & $5(41.66 \%)$ \\
Personal history of atopy & $2(5.26 \%)$ & $11(39.28 \%)$ & $6(50 \%)$ \\
Koebner's phenomenon & & &
\end{tabular}

Group B (stage I vitiligo) Twenty eight patients with stage I vitiligo (hypopigmented areas with partial pigment loss) were included in this study with mean age \pm SD was $15.46 \pm 12.50$ years with 15 (57.14\%) males patients and 12 (42.86\%) females patients. The duration of disease was less than 1 year in 14 (50\%) patients and from $1-5$ years in $10(35.71 \%)$ patients and more than 5 years in $4(14.29 \%)$ patients. The number of patients with a topy was $6(21.43 \%)$ patients. The family history of vitiligo was found in 11 (39.29\%) patients and the family history of other autoimmune disease was found in 8 (28.57\%) patients. Koebner's phenomenon was positive in $11(39.28 \%)$ patients (Table 1 ).

\section{Group C (Combination of pityriasis alba and vitiligo patients)}

Twelve patients were included in this study all of them had pityriasis alba and vitiligo in the same patient (Figure 3), with mean age \pm SD was $8.33 \pm 2.78$ years including $9(75 \%)$ males patients and $3(25 \%)$ females patients. The duration of pityriasis alba was ranged from 2 weeks to 8 years, the patients less than 1 years were $3(25 \%)$ and from 1 - 5 years in $6(50 \%)$ patients and more than 5 years in $3(25 \%)$ patients. While duration of vitiligo less than 1 year was in $9(75 \%)$ patients while more than 1 year was in $3(33.33 \%)$ patients. Vitiligo was active in 9 (75\%) patients while Koebner's phenomenon was positive in $6(50 \%)$ patients (Table 1).

\subsection{Histopathological Study}

Group A (Pityriasis alba)

Hematoxylin-Eosin (H \& E) stain: epidermal lymphocytic infiltration was 
observed in $2(5.26 \%)$ patients. Regarding melanin stores, basal melanin was found to be decreased when compared with normal skin in 23 (60.53\%) patients and focal decrease in basal melanin in $13(34.21 \%)$ patients and normal basal melanin in $2(5.26 \%)$ patients while no melanophages were detected. Perivascular and Periappendiceal lymphocytic infiltration was mild in 20 (52.63\%) patients and moderate in 12 (31.58\%) patients and severe in 6 (15.79\%) patients.

Fontana Masson stain showed decrease in basal melanin when compared with normal skin in $24(63.16 \%)$ patients and focal decrease in $11(28.95 \%)$ patients and normal melanin in $3(7.89 \%)$ patients. While melanophages were present in 2 (5.26\%) patients (Figure 4 and Figure 5, Table 2).

Group B (stage I vitiligo patients);

Hematoxylin-Eosin ( $\mathrm{H} \& \mathrm{E}$ ) stain: epidermal lymphocytic infiltration was seen in $5(17.86 \%)$ patients. Regarding melanin store, there was partial basal melanin loss in 19 (67.86\%) patients, focal decrease in 7 (25\%) patients and no basal melanin in $2(7.14 \%)$ patients and as compared with normal skin. Perivascular infiltration was seen as a mild degree in $14(50 \%)$ patients and moderate in $10(35.71 \%)$ patients and severe in $4(14.29 \%)$ patients. Melanophages were present in $3(10.71 \%)$ patients and degeneration of collagen and high fibroblast number were observed in $1(3.57 \%)$ patient. Fontana stain showed partial melanin loss in $20(71.43 \%)$ patients and focal decrease in $8(28.57 \%)$ patients while melanophages were present in 3 (10.71\%) patients (Figure 6, Table 2).

\section{Group C: (Combination of pityriasis alba and vitiligo patients)}

\section{Hematoxylin-Eosin (H \& E) stain}

Vitiligo lesion showed epidermotropism in 2 (16.67\%) while basal melanin was absent in $4(33.33 \%)$ patients and $6(50 \%)$ patients showed decrease in basal melanin and focal decrease in $2(16.67 \%)$ patients as compared with normal skin. Perivascular infiltration was seen as mild in $3(25 \%)$ patients and moderate in $2(16.67 \%)$ patients and severe in $7(58.33 \%)$ patients. Fontana stain showed decrease in melanin in basal layer in $8(66.67 \%)$ and focal decrease in $1(8.33 \%)$ patient and absent in $3(25 \%)$ patients, while no melanophages were observed

In pityriasis alba, the basal melanin was partially decreased in $8(66.67 \%)$ patients while focal decrease was seen in $4(33.33 \%)$ patients. The Perivascular infiltration was mild in $4(33.33 \%)$ patients and moderate in $5(41.67 \%)$ patients and severe in $3(25 \%)$ patients. Fontana stain showed partial loss of melanin in $6(50 \%)$ patients and focal decrease in $6(50 \%)$ patients while melanophages were detected in $2(16.67 \%)$ patients (Table 3).

The $\mathrm{p}$-value estimated by chi square for comparison between three groups was showed that the p-value between group A (pityriasis alba) and group B (stage I vitiligo) in basal melanin in $\mathrm{H} \& \mathrm{E}$ stain was 0.540 and in Fontana stain was 0.692 mean there was no statistical significant deference between two groups (Table 4 and Table 5) while between pityriasis alba lesion and vitiligo lesion on group $\mathrm{C}$ in $\mathrm{H} \& \mathrm{E}$ stain was 0.345 and in Fontana stain was 0.407 and also there was no statistically significant difference (Table 6 and Table 7). 
Table 2. Thehistopathological finding in group A (pityriasis alba) \& B (stage I vitiligo).

\begin{tabular}{ccc}
\hline Histopathological changes & $\begin{array}{c}\text { Pityriasis alba } \\
(\mathbf{A})\end{array}$ & $\begin{array}{c}\text { Vitiligo } \\
(\mathbf{B})\end{array}$ \\
\hline Epidermal changes Acanthosis & $32(84.21 \%)$ & $17(60.71 \%)$ \\
Basal liquefaction & $4(10.52 \%)$ & $2(7.14 \%)$ \\
Epidermotropism & $2(5.26 \%)$ & $5(17.86 \%)$ \\
Inflammatory reaction & & \\
Mild & $20(52.63 \%)$ & $14(50 \%)$ \\
Moderate & $12(31.58 \%)$ & $10(35.71 \%)$ \\
Sever & $6(15.79 \%)$ & $4(14.29 \%)$ \\
Basal melanin & & $7(25 \%)$ \\
Focal decrease & $13(34.21 \%)$ & $19(67.86 \%)$ \\
Partial all field decrease & $23(60.53 \%)$ & $0 \%$ \\
Normal melanin & $2(5.26 \%)$ & $2(7.14 \%)$ \\
No basal melanin & $0 \%$ & $3(10.71 \%)$ \\
Melanophages & $2(5.26 \%)$ & \\
Melanin in Fontana & & $8(28.57 \%)$ \\
Focal decrease & $11(28.95 \%)$ & $20(71.43 \%)$ \\
Partial decrease & $24(63.16 \%)$ & $0 \%$ \\
Normal melanin & $3(7.89 \%)$ & \\
\hline
\end{tabular}

Table 3. The histopathological finding in group C (combination group pityriasis alba and vitiligo).

\begin{tabular}{ccc}
\hline Histopathological change & Pityriasis alba lesion & Vitiligo lesion \\
\hline Epidermal changes & & \\
Acanthosis & $9(75 \%)$ & $9(75 \%)$ \\
Basal liquefaction & $1(8.33 \%)$ & $0 \%$ \\
Epidermotropism & $0 \%$ & $2(16.67 \%)$ \\
Inflammatory reaction & & \\
Mild & $4(33.33 \%)$ & $3(25 \%)$ \\
Moderate & $5(41.67 \%)$ & $2(16.67 \%)$ \\
Sever & $3(25 \%)$ & $7(58.33 \%)$ \\
Basal melanin & & $2(16.67 \%)$ \\
Focal decrease & $4(33.33 \%)$ & $6(50 \%)$ \\
partial) decrease & $8(66.67 \%)$ & $4(33.33 \%)$ \\
No melanin & $0 \%$ & $0 \%$ \\
Melanophage & $0 \%$ & $1(8.33 \%)$ \\
Melanin in Fontana & & $8(66.67 \%)$ \\
Focal decrease & $6(50 \%)$ & $3(25 \%)$ \\
Partial decrease & $6(50 \%)$ & \\
No melanin & $0 \%$ & \\
\hline
\end{tabular}

Table 4. Comparison of basal melanin in H \& E stainbetween group A (pityriasis alba) \& $\mathrm{B}$ (stage I vitiligo) according to compare with normalskin under microscope examination and using chi square to calculate $\mathrm{p}$-value.

\begin{tabular}{ccccccc}
\hline Groups & $\begin{array}{c}\text { Normal basal } \\
\text { melanin }\end{array}$ & $\begin{array}{c}\text { No basal } \\
\text { melanin }\end{array}$ & $\begin{array}{c}\text { Partial decrease Focal decrease } \\
\text { in all field }\end{array}$ & in melanin & Total & P-value \\
\hline Group A & $2(5.26 \%)$ & $0 \%$ & $23(60.53 \%)$ & $13(34.21 \%)$ & $38(100 \%)$ & \\
Group B & $0 \%$ & $2(7.14 \%)$ & $19(67.86 \%)$ & $7(25 \%)$ & $28(100 \%)$ & 0.540 \\
Total & 2 & 2 & 42 & 20 & 66 & \\
\hline
\end{tabular}


Table 5. Comparison of melanin in fontana stainbetween group A (pityriasis alba) \& B (stage I vitiligo) according to compare with normal skin under microscope examination and using chi square to calculate p-value.

\begin{tabular}{cccccc}
\hline Groups & $\begin{array}{c}\text { Normal basal } \\
\text { melanin. }\end{array}$ & $\begin{array}{c}\text { Partial decrease } \\
\text { in melanin }\end{array}$ & $\begin{array}{c}\text { Focal decrease } \\
\text { in melanin }\end{array}$ & Total & P-Value \\
\hline Group A & $3(7.89 \%)$ & $24(63.16 \%)$ & $11(28.95 \%)$ & $38(100 \%)$ & 0.692 \\
Group B & $0(0 \%)$ & $19(67.86 \%)$ & $9(23.95 \%)$ & $28(100 \%)$ & \\
Total & 3 & 43 & 20 & 66 & \\
\hline
\end{tabular}

Table 6. Comparison of basal melanin in $\mathrm{H}$ \& E stainbetween pityriasis alba lesion and vitiligo lesion in group $\mathrm{C}$ (combination group) according to compare with normal skin under microscope examinationand using chi square to calculate p-value.

\begin{tabular}{cccccc}
\hline Group C & $\begin{array}{c}\text { No basal } \\
\text { melanin }\end{array}$ & $\begin{array}{c}\text { All field decrease } \\
\text { in melanin }\end{array}$ & $\begin{array}{c}\text { Focal decrease } \\
\text { in melanin }\end{array}$ & Total & P-value by \\
\hline Pityriasis alba & $0(0 \%)$ & $8(66.67)$ & $4(33.33 \%)$ & $12(100 \%)$ & 0.345 \\
Vitiligo & $4(33.33 \%)$ & $6(50 \%)$ & $2(16.67 \%)$ & $12(100 \%)$ & \\
Total & 4 & 14 & 6 & 24 & \\
\hline
\end{tabular}

Table 7. Comparison of basal melanin in fontana stainbetween pityriasis alba lesions and vitiligo lesions in group $\mathrm{C}$ (combination group) according to compare with normal skin under microscope examination and using chi square to calculate $\mathrm{p}$-value.

\begin{tabular}{cccccc}
\hline GroupC & $\begin{array}{c}\text { No basal } \\
\text { melanin }\end{array}$ & $\begin{array}{c}\text { Partial } \\
\text { decrease }\end{array}$ & $\begin{array}{c}\text { Focal } \\
\text { decrease }\end{array}$ & Total & P-value \\
\hline Pityriasis alba & $0(0 \%)$ & $6(50 \%)$ & $6(50 \%)$ & $12(100 \%)$ & 0.407 \\
Vitiligo & $3(25 \%)$ & $8(66.67 \%)$ & $1(8.33 \%)$ & $12(100 \%)$ & 24 \\
Total & 3 & 18 & 7 & \\
\hline
\end{tabular}

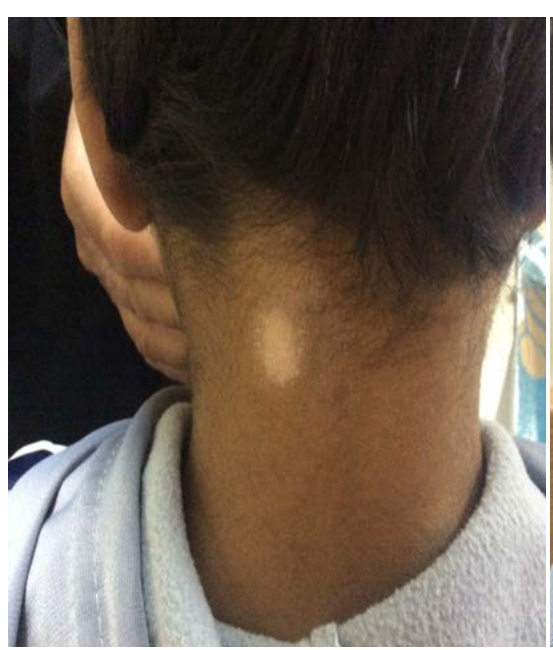

(a)

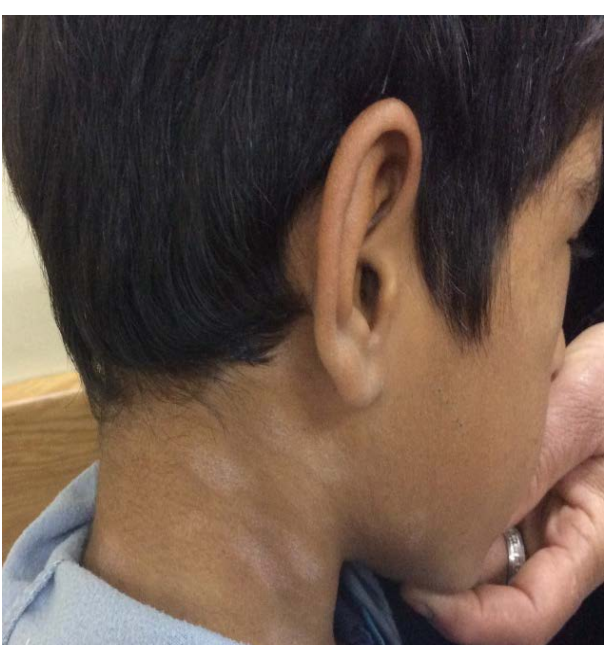

(b)

Figure 3. Six years old male with history of Pityriasis alba for 3 years that progressed into vitiligo on same site after eighteen months. 


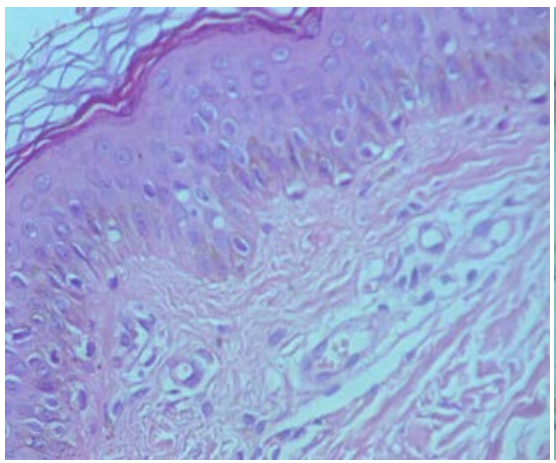

(a)

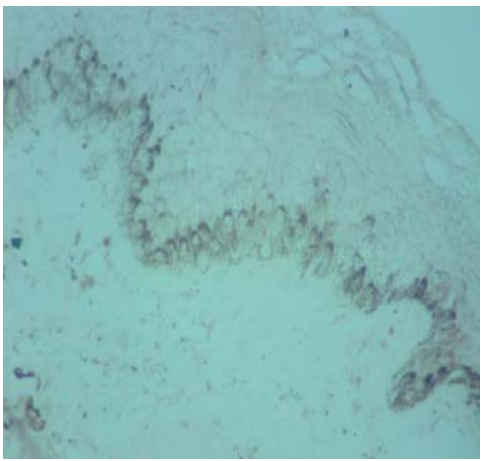

(b)

Figure 4. Normal skin from ten years old male and biopsy was taken from his arm (a) histopathology using $\mathrm{H} \& \mathrm{~S}$ stain $(\times 40)$ showed normal histology and (b) Fontana stain $(\times 40)$ showed normal basal melanin.

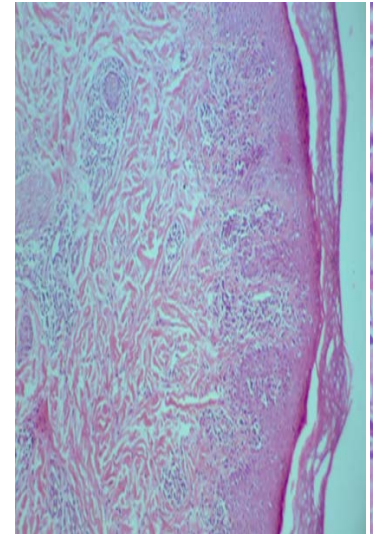

(a)

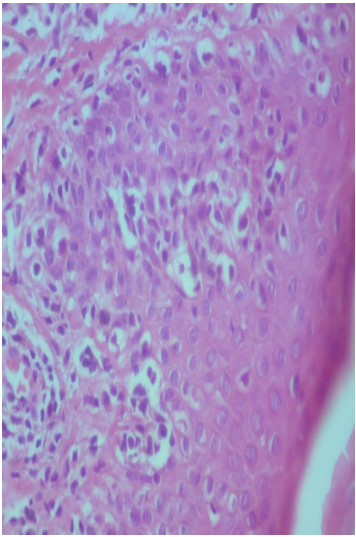

(b)

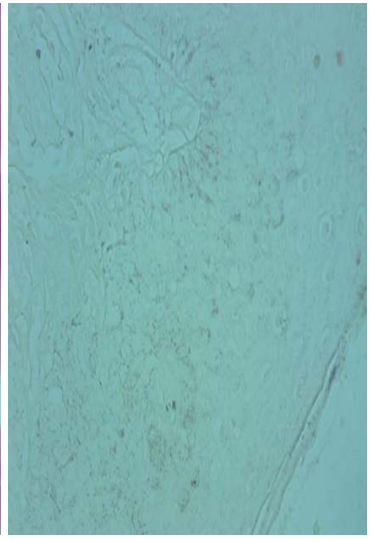

(c)

Figure 5. Five years old male with Pityriasis alba for months (a) Histopathology stained with $\mathrm{H} \& \mathrm{E}(10 \times)$ showedacanthosissuperficialperivascular inflammatory reaction, and decrease in basal melanin; (b) (40x) showed inflammatory lymphocytic infiltrateof epidermis and decrease in basal melanin; (c) Fontana stain $(40 \times)$ showed reduction in basal melanin.

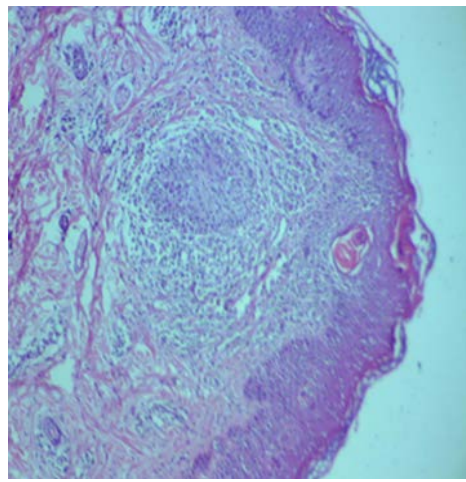

(a)

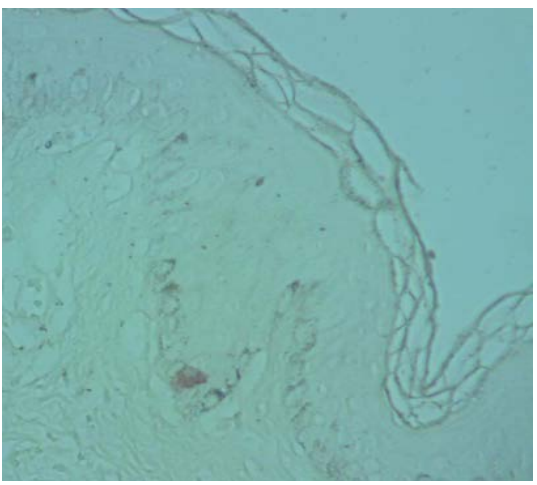

(b)

Figure 6. Eight years old male with vitiligo for 3 years and biopsy was taken from trunk (a) histopathology $\mathrm{H}$ \& $\mathrm{E}$ stain $(10 \times)$ showed acanthosis, perivascular and periappendagealinflammatory infiltration and decrease in basal melanin; (b) Fontana stain $(40 \times)$ showed reduction in basal melanin. 


\section{Discussion}

Recently Sharquie et al. published as a study showed that pityriasis alba might progress into vitiligo during course of disease by the following observations: (43.75\%) of patients showed progression of their original pityriasis alba lesion into vitiligo, also $34.35 \%$ of patients had positiveKoebner's phenomenon and $31.25 \%$ of patients had positive family of vitiligo [4].

So the target of present work is to confirm this hypothesis. Histopathology of stage I vitiligo had similarities with pityriasis alba as in stage I vitiligo, the rash of the disease appeared light brown hypopigmention in which there was partialpigment loss with inflammatory reaction. Epidermal mononuclear cell infiltration was seen in $80 \%$ of both the marginal areas and stage I vitiligo specimens [5]. While Histopathology of pityriasis alba was Perivascular lymphocytic infiltrates and occasionally seen in the epidermisand Fontana-Masson staining showed that the amount of epidermal melanin was found to be significantly decreased in the epidermis of skinlesion when compared with perilesional normal skin [8].

While the present work had showed the following main findings: family history of vitiligo was positive in pityriasis albain $44.74 \%$, stage I vitiligo was in $39.29 \%$ and in combination of pityriasis alba and vitiligo was $58.33 \%$ and this was in favor that both diseases were sharing common genetic element. While Koebner's phenomenon was positive in all 3 groups as seen in $5.26 \%$ of pityriasis alba and $39.28 \%$ in stage I vitiligo patients and in combination group was positive in $50 \%$ of patients. These findings strongly support the theory that vitiligo might start as pityriasis alba.

While the histopathology of the three groups showed inflammatory reaction in epidermis and dermis, melanin stores were reduced in the epidermis especially in the basal layered in all groups while melanophages were detected in dermis of patients with vitiligo and pityriasis alba.

\section{Conclusion}

The findings that are observed in present study are strongly in favor of the hypothesis that says pityriasis alba and vitiligo are sharing one genetic, clinical and histopathological pictures. As positive family history of vitiligo and high Koebner's phenomenon are seen in all three groups. Also there are many histopathological and histochemical similarities in these two diseases.

\section{Conflicts of Interest}

The authors declare no conflicts of interest regarding the publication of this paper.

\section{References}

[1] Wolff, K., Goldsmith, L.A. and Katz, S.I. (2012) Acquired Localized Hypomelanoses. In: Fitzpatrick's Dermatology in General Medicine, 7th Edition, McGraw-Hill 
Company, New York, 807-808.

[2] Holden, C.A. and Berth-Jones, J. (2010) Eczema, Lichenification, Prurigo and Erythroderma. In: Burns, T., Breathnach, S. and Cox, N., Eds., Rook's Textbook of Dermatology, Blackwell Publishing, Oxford, 23-27.

[3] Sharquie, K.E. (2011) Common Hypopigmented Skin Disorder in Baghdad Teaching Hospital. Iraqi Journal of Community Medicine, 24, 258-260.

[4] Sharquie, K.E., Noaimi, A.A. and Salmo, H.M. (2013) Pityriasis Alba versus Vitiligo. Journal of Saudi Society of Dermatology and Dermatological Surgery, 17, 51-54. https://doi.org/10.1016/j.jssdds.2013.05.002

[5] Sharquie, K.E., Mehenna, S.H., Naji, A.A. and Al-Azzawi, H. (2004) Inflammatory Changes in Vitiligo: Stage I and II Depigmentation. The American Journal of Dermatopathology, 26, 108-112. https://doi.org/10.1097/00000372-200404000-00004

[6] Rook, M.A.J. (1971) Vitiligo with Raised Rim in Atopic Subjects. BJD, 85, 491. https://doi.org/10.1111/j.1365-2133.1971.tb14062.x

[7] Sharquie, K.E. (1982) The Histology and Immunopathology of Vitiligo. University of Sheffield, Sheffield.

[8] In, S.I., Kang, H.Y. and Lee, E.-S. (2008) Clinical and Histopathological Characteristics of Pityriasis Alba. Clinical and Experimental Dermatology, 34, 1-7. 